This is the final peer-reviewed accepted manuscript of:

Cinti, E., Davila, J. and Del Pino, M. (2016), Solutions of the fractional Allen-Cahn equation which are invariant under screw motion. Journal of the London Mathematical Society, 94: 295-313.

The final published version is available online at: https://doi.org/10.1112/jlms/jdw033

Rights / License:

The terms and conditions for the reuse of this version of the manuscript are specified in the publishing policy. For all terms of use and more information see the publisher's website.

This item was downloaded from IRIS Università di Bologna (https://cris.unibo.it/)

When citing, please refer to the published version. 


\title{
SOLUTIONS OF THE FRACTIONAL ALLEN-CAHN EQUATION WHICH ARE INVARIANT UNDER SCREW MOTION
}

\author{
ELEONORA CINTI, JUAN DÁVILA, AND MANUEL DEL PINO
}

Abstract. We establish existence and non-existence results for entire solutions to the fractional Allen-Cahn equation in $\mathbb{R}^{3}$, which vanish on helicoids and are invariant under screw-motion. In addition, we prove that helicoids are surfaces with vanishing nonlocal mean curvature.

\section{INTRODUCTION}

In this paper we establish existence results for a class of entire solutions to the fractional Allen-Cahn equation

$$
(-\Delta)^{\alpha} u+F^{\prime}(u)=0 \text { in } \mathbb{R}^{n},
$$

where $F$ is a double-well potential, i.e. it satisfies the following properties:

- $t \mapsto F(t)$ is an even, positive function of class $C^{2, \gamma}$, with $\gamma>$ $\max \{0,1-2 \alpha\}$,

- $F(t) \geq F( \pm 1)$ and equality holds if and only if $t= \pm 1$.

Moreover, we assume also that

$$
F^{\prime \prime}(0)<0 \text { and } F^{\prime \prime}(0) t \leq F^{\prime}(t) \text { for every } t \geq 0 .
$$

A classical example of such potential is $F(t)=\frac{1}{4}\left(1-t^{2}\right)^{2}$.

The fractional Laplacian $(-\Delta)^{\alpha}$ is a pseudodifferential operator with symbol $|\xi|^{2 \alpha}$ (where $\xi$ is the variable in the frequency space, $\alpha \in(0,1)$ ), and it can also be defined in the following way:

$$
(-\Delta)^{\alpha} u(x):=C_{n, \alpha} P . V . \int_{\mathbb{R}^{n}} \frac{u(x)-u(y)}{|x-y|^{n+2 \alpha}} d y,
$$

where P.V. stands for the Cauchy principal value and $C_{n, \alpha}$ denotes a constant depending only on $n$ and $\alpha$.

In the last years, there has been much interest in the study of solutions to the fractional Allen-Cahn equation. In [8, 9, 10, 21], the existence of layer-type solutions, that is solutions monotone in one direction with limits \pm 1 at $\pm \infty$, has been established, while in [14], one of the authors proved

2010 Mathematics Subject Classification. Primary: 35J61, 35J20; Secondary: 35B08, $47 \mathrm{~J} 30$.

Key words and phrases. Fractional Laplacian, entire solutions, nonlocal minimal surfaces. 
existence for saddle-shaped solution, that are solutions which vanish on the Simons cone

$$
\mathcal{C}=\left\{(x, \xi) \in \mathbb{R}^{m} \times \mathbb{R}^{m}:|x|=|\xi|\right\},
$$

they are odd with respect to $\mathcal{C}$ and even with respect to the coordinate axis. In all these works, the proof of existence relies on a variational argument, which makes use of the symmetries of the problem. Also in this paper we are interested in solutions of (1.1) which satisfies some symmetry properties, and the technique we use relies on the variational structure of (1.1).

More precisely we establish existence and non-existence results for solutions which vanish on helicoids and are invariant under screw motion (see (1.4) and (1.5) below for precise definitions). For the classical Allen-Cahn equation, analogue results are contained in a work by Musso, Pacard and one of the authors [18].

The interest in the study of solutions which vanish on helicoids comes from the fact that helicoids are surfaces with zero mean curvature.

There is a very strict connection between the Allen-Cahn equation and the classical theory of minimal surfaces. The classical result by Modica and Mortola [20] establishes that the energy functional associated to the classical Allen-Cahn equation, after a suitable rescaling, $\Gamma$-converges to the Perimeter functional.

In the fractional setting, an analougue $\Gamma$-convergence type result has been established in [1, 19] for powers $1 / 2 \leq \alpha<1$, and in [22] for any power $0<\alpha<1$ : after a suitable rescaling, the energy functional associated to the fractional Allen-Cahn equation $\Gamma$-converges to the classical perimeter if $1 / 2 \leq \alpha<1$ and to the nonlocal perimeter if $0<\alpha<1 / 2$.

This deep relation betwewn the thoery of minimal surfaces and the theory of phase transitions is also one of the main motivation to the well known Conjecture of De Giorgi about one-dimensional symmetry of bounded monotone solutions to the Allen-Cahn equation. In the fractional setting this problem has been studied in several works (see $[4,5,9,10]$ ).

The notion of nonlocal perimeter has been introduced by Caffarelli, Roquejoffre and Savin in [12], where existence, regularity results and a monotonicity formula for nonlocal minimal surfaces have been established. Similarly to the case of the classical perimeter, performing the first variation of the nonlocal perimeter functional, one can define the notion of nonlocal mean curvature (see (1.10) below).

In this paper, we focus our attention both on the PDE problem and on the geometric one. Indeed in Theorems 1.1 and 1.2 below we establish existence and non-existence results for solutions to (1.1) (in the case of space dimension $n=3$ ) which vanish on helicoids and are invariant under screw motion. Moreover, in Theorem 1.3 we prove that helicoids have zero nonlocal mean curvature.

We recall now the definition of helicoid and screw motion. We will work in dimension $n=3$. Given $\lambda>0$, the helicoid $H_{\lambda}$ is the minimal surface 
which can be parametrized in the following way

$$
\mathbb{R} \times \mathbb{R} \ni(t, \theta) \mapsto\left(t e^{i \theta}, \frac{\lambda}{\pi} \theta\right) \in \mathbb{C} \times \mathbb{R}=\mathbb{R}^{3} .
$$

The screw motion of parameter $\lambda$ acting on $\mathbb{C} \times \mathbb{R}$ is given by

$$
\sigma_{\lambda}^{\beta}(z, s)=\left(e^{i \beta} z, s+\frac{\lambda}{\pi} \beta\right) .
$$

Obviously $H_{\lambda}$ is invariant under the action of $\sigma_{\lambda}^{\beta}$ for every $\beta \in \mathbb{R}$.

Our first main result is the construction of a nontrivial entire solution to (1.1) in dimension 3 which vanishes on $H_{\lambda}$, provided $\lambda$ is chosen sufficiently large. We define

$$
\lambda_{*}:=\frac{\pi}{\left(-F^{\prime \prime}(0)\right)^{\frac{1}{2 \alpha}}} .
$$

Theorem 1.1. Let $n=3$. Assume that $F$ is a double-well potential satisfying (1.2) and that $\lambda>\lambda_{*}$.

Then, there exists a solution of the fractional Allen-Cahn equation (1.1) whose zero set is equal to $H_{\lambda}$. This solution is invariant under the screw motion of parameter $\lambda$, i.e.

for every $\beta \in \mathbb{R}$.

$$
u \circ \sigma_{\lambda}^{\beta}=u
$$

In the following result, we also prove that Theorem 1.1 is, in some sense, sharp.

Theorem 1.2. Let $n=3$. Assume that $F$ is a double-well potential satisfying (1.2) and that $\lambda \leq \lambda_{*}$.

Then, there are no nontrivial bounded solutions of (1.1), which vanish on the helicoid $H_{\lambda}$ and are invariant under the screw motion of parameter $\lambda$.

To prove the previous results, we will realize the nonlocal problem (1.1) as a local problem in $\mathbb{R}_{+}^{n+1}$ with a nonlinear Neumann condition on $\partial \mathbb{R}_{+}^{n+1}=\mathbb{R}^{n}$ (the so called Caffarelli-Silvestre extension [13]).

More precisely, if $u=u(x)$ is a function defined on $\mathbb{R}^{n}$, we consider its s-harmonic extension $v=v(x, y)$ in $\mathbb{R}_{+}^{n+1}=\mathbb{R}^{n} \times(0,+\infty)$. It is well known (see $[10,13])$ that $u$ is a solution of $(1.1)$ if and only if $v$ satisfies

$$
\begin{cases}\operatorname{div}\left(y^{1-2 \alpha} \nabla v\right)=0 & \text { in } \mathbb{R}_{+}^{n+1}, \\ -\frac{1}{c_{\alpha}} \lim _{y \rightarrow 0} y^{1-2 \alpha} \partial_{y} v=-F^{\prime}(v) & \text { on } \mathbb{R}^{n}=\partial \mathbb{R}_{+}^{n+1},\end{cases}
$$

where $c_{\alpha}$ is the constant given in (2.3).

The energy associated to problem (1.7) is

$$
E(v)=\frac{1}{2 c_{\alpha}} \int_{0}^{+\infty} \int y^{1-2 \alpha}|\nabla v|^{2} d x d y+\int F(v(x, 0)) d x .
$$

Both proofs of Theorems 1.1 and 1.2 follows the ideas contained in [18] for the local case. 
Using the symmetries of the problem, the proof of existence for solutions to the problem (1.7) above, is based on an existence result for an analogous problem in the 2 -dimensional cylinder $[0, \lambda] \times(0,+\infty)$ with zero-Dirichlet boundary conditions on the lateral boundary $\partial[0, \lambda] \times(0,+\infty)$. Existence and nonexistence results for this 2-dimensional problem will be studied in Section 3 (see Lemma 3.1). Here we emphasize that the length $\lambda$ of the interval coincides with the parameter of the helicoid $H_{\lambda}$.

Another important tool in the proof of the existence result Theorem 1.1 will be an energy estimate for minimizers, which will ensures us that the limit of a minimizing sequence does not identically vanish. In this step we follows the technique used in [14], based on a suitable choice of a cut-off function.

On the other hand, for the non-existence result, a crucial ingredient will be an exponential decay in the $y$-variable of the extended solution $v$ of (1.7), under our symmetry assumption (see Proposition 5.1 below).

We recall now the notions of nonlocal minimal surface and of nonlocal mean curvature. Nonlocal minimal surfaces were introduced in [12] as boundaries of measurable sets $E$ whose characteristic function $\chi_{E}$ minimizes an $H^{\alpha}$-norm. More precisely, for any $0<\alpha<1 / 2$, the nonlocal $\alpha$-perimeter functional of a set $E$ in an open set $\Omega \subset \mathbb{R}^{n}$, is given by

$$
\operatorname{Per}_{2 \alpha}(E, \Omega):=L\left(E \cap \Omega, \mathbb{R}^{n} \backslash E\right)+L(E \backslash \Omega, \Omega \backslash E),
$$

where, for two disjoint measurable sets $A$ and $B, L(A, B)$ denotes the quantity

$$
L(A, B):=\int_{A} \int_{B} \frac{1}{|x-\bar{x}|^{n+2 \alpha}} d x d \bar{x} .
$$

A set $E$ is said to be $\alpha$-minimal in $\Omega$ if

$$
\operatorname{Per}_{2 \alpha}(E, \Omega) \leq \operatorname{Per}_{2 \alpha}(F, \Omega)
$$

for any measurable set $F$ with $E \triangle F \subset \subset \Omega$. Notice that in the literature (see e.g. [12]) the fractional $s$-perimeter is defined for any $s \in(0,1)$ and corresponds to (1.9) for $s=2 \alpha$. Here, we prefer to keep this notation to be consistent with the fractional power of the Laplacian.

Analogously to the classical theory of minimal surfaces, performing the first variation of the nonlocal perimeter functional, we end up with the notion of nonlocal mean curvature. More precisely, the Euler-Lagrange equation for $\operatorname{Per}_{2 \alpha}\left(E, \mathbb{R}^{n}\right.$ ) (on the boundary of $E$ ) is given by

$$
\mathcal{H}_{\partial E}^{2 \alpha}(x):=\int_{\mathbb{R}^{n}} \frac{\chi_{E}(\bar{x})-\chi_{E^{C}}(\bar{x})}{|x-\bar{x}|^{n+2 \alpha}} d \bar{x}=0 \quad \text { for any } x \in \partial E,
$$

where $E^{C}:=\mathbb{R}^{n} \backslash E$ and $\mathcal{H}_{\partial E}^{2 \alpha}$ denotes the nonlocal mean curvature of the boundary of $E$ (we write NMC for short).

Recently, there has been much interest in the study of surfaces with vanishing or constant nonlocal mean curvature. In [17], two of the authors and J. Wei provide examples of surfaces with zero NMC. More precisely, they 
establish existence of nonlocal minimal Lawson cones (for any $0<\alpha<1 / 2$ ) and proved their stability in dimension 7 for $\alpha$ small. Moreover, for $\alpha \rightarrow 1 / 2$, they constructed the nonlocal analogue of catenoids. Concerning the study of surfaces with constant NMC, in $[7,15]$ the analogue of Alexandrov Theorem, characterizing spheres as the only closed embedded hypersurfaces in $\mathbb{R}^{n}$ with constant mean curvature, has been established. In $[7,6]$ the existence of Delaunay-type surfaces is established, while in [16] periodic and cylindrical symmetric hypersurfaces, which minimize a certain fractional perimeter under a volume constraint, are considered.

In our last result we provide a new example of surface with zero NMC. Using the fact that the (double) helicoid $H_{\lambda}$ can be seen as the boundary of a set $E_{+}$(as it will be made precise in (6.1)), we prove that helicoids, which have zero (classical) mean curvature, also have zero NMC.

Theorem 1.3. For any $\lambda>0$ and for any $0<\alpha<1 / 2$, we have that

$$
\mathcal{H}_{H_{\lambda}}^{2 \alpha} \equiv 0
$$

The paper is organized as follows:

- in Section 2, we recall some preliminaries on the fractional Laplacian in a bounded domain with 0-Dirichlet boundary condition.

- in Section 3, we establish existence and non existence results for a fractional Dirichlet problem in the 1-dimensional case.

- in Section 4, we prove our existence result Theorem 1.1.

- in Section 5, we give the proof of Theorem 1.2.

- in Section 6, we prove Theorem 1.3.

\section{Preliminaries}

As mentioned in the Introduction, thanks to the symmetries of our problem, the proof of our existence result Theorem 1.1 is based on an existence result for the following two-dimensional problem

$$
\begin{cases}\operatorname{div}\left(y^{1-2 s} \nabla v(s, y)\right)=0 & \text { for }(s, y) \in[0, \lambda] \times(0,+\infty) \\ v(s, y)=0 & \text { for }(s, y) \in \partial[0, \lambda] \times(0,+\infty) \\ -\frac{1}{c_{\alpha}} \lim _{y \rightarrow 0} y^{1-2 \alpha} \partial_{y} v=-F^{\prime}(v) & \text { on }[0, \lambda] \times\{y=0\}\end{cases}
$$

which we will establish in Lemma (3.1) below.

As we will clarify later on in this section, this is the extended problem associated to a fractional one-dimensional problem with zero-Dirichlet boundary condition in the interval $[0, \lambda]$. Hence, in particular, the existence result of Lemma 3.1 implies an existence result for solutions of a fractional Dirichlet problem on an interval $[0, \lambda]$.

For the sake of clarity and completeness, in this section we collect some well known facts about the fractional Laplacian in a bounded domain (see $[2,11])$ with zero-Dirichlet boundary condition. The results contained in this section will be used later on in Section 3 and in Section 5, where we will use in particular the exponential decay (2.11). 
Let $\Omega$ be a sufficiently regular (say Lipschitz) domain in $\mathbb{R}^{n}$. We denote by $A_{\alpha}$ the fractional power of the Laplacian in $\Omega$ with zero Dirichlet boundary condition on $\partial \Omega$.

To define $A_{\alpha}$, let us consider $\left\{\mu_{k}, \zeta_{k}\right\}_{k=1}^{\infty}$ the eigenvalues and corresponding eigenfunctions of the Laplacian $-\Delta$ in $\Omega$ with zero Dirichlet boundary condition:

$$
\begin{cases}-\Delta \zeta_{k}=\mu_{k} \zeta_{k} & \text { in } \Omega \\ \zeta_{k}=0 & \text { on } \partial \Omega\end{cases}
$$

Let $u=\sum_{k=1}^{\infty} a_{k} \zeta_{k}$, then we define

$$
A_{\alpha} u=\sum_{k=1}^{\infty} a_{k} \mu_{k}^{\alpha} \zeta_{k} .
$$

We stress that the operator $A_{\alpha}$ defined above is different from the operator $(-\Delta)^{\alpha}$ defined in (1.3), as it is shown in [23], where the two operators have been compared with particular reference to their spectrum.

Let now $\mathcal{C}_{\Omega}$ be the cylinder $\mathcal{C}_{\Omega}=\Omega \times(0, \infty)$ and $\partial_{L} \mathcal{C}_{\Omega}=\partial \Omega \times(0, \infty)$ its lateral boundary. Following $[2,11]$, we can consider the extension operator with zero Dirichlet boundary condition on the all $\partial_{L} \mathcal{C}_{\Omega}$.

Definition 2.1. We define the $\alpha$-harmonic extension $v=\mathcal{E}_{\alpha}(u)$ in $\mathcal{C}_{\Omega}$ of a function $u$ defined in $\Omega$ and vanishing on $\partial \Omega$ as the solution of the problem

$$
\begin{cases}\operatorname{div}\left(y^{1-2 \alpha} \nabla v\right)=0 & \text { in } \mathcal{C}_{\Omega} \\ v=0 & \text { on } \partial_{L} \mathcal{C} \\ v=u & \text { on } \Omega \times\{y=0\} .\end{cases}
$$

It is well known that (see $[2,11])$

$$
A_{\alpha} u(x)=-\frac{1}{c_{\alpha}} \lim _{y \rightarrow 0} y^{1-2 \alpha} \partial_{y} v(x, y)
$$

where

$$
c_{\alpha}=\frac{2^{1-2 \alpha} \Gamma(1-\alpha)}{\Gamma(\alpha)} .
$$

We recall now the explicit expression (see Lemma 3.4 in [2]) for $\mathcal{E}_{\alpha}(u)$ in terms of the spectral decomposition (2.1).

Lemma 2.2 (Lemma 3.4 in [2]). Let $\left\{\mu_{k}, \zeta_{k}\right\}$ be, as before, the eigenvalues and eigenfunctions of $-\Delta$ in $\Omega$ (with zero Dirichlet boundary condition). Let $u=\sum_{k=1}^{\infty} a_{k} \zeta_{k}$ be such that $\sum_{k=1}^{\infty} a_{k} \mu_{k}^{\alpha}<\infty$. Then, the $\alpha$-harmonic extension of $u$ is given by

$$
v(x, y)=\mathcal{E}_{\alpha}(u)(x, y)=\sum_{k=1}^{\infty} a_{k} \zeta_{k}(x) \varphi\left(\mu_{k}^{\frac{1}{2}} y\right),
$$


where $\varphi$ is a solution of the problem

$$
\left\{\begin{array}{l}
\varphi^{\prime \prime}+\frac{1-2 \alpha}{y} \varphi^{\prime}-\varphi=0 \quad \text { for } y>0 \\
-\lim _{y \rightarrow 0} y^{1-2 \alpha} \varphi^{\prime}(y)=c_{\alpha} \\
\varphi(0)=1
\end{array}\right.
$$

The solution $\varphi$ coincides with the solution of the following problem

$$
\varphi^{\prime \prime}+\frac{1-2 \alpha}{y} \varphi^{\prime}-\varphi=0, \quad \varphi(0)=1, \quad \lim _{y \rightarrow \infty} \varphi(y)=0,
$$

and minimizes the functional

$$
\int_{0}^{\infty} y^{1-2 \alpha}\left(|\varphi(y)|^{2}+\left|\varphi^{\prime}(y)\right|^{2}\right) d y
$$

Moreover, it is a combination of Bessel functions, as shown in the following lemma.

Lemma 2.3 (Lemma 2.2 in [3]). The solution of the $O D E$

$$
\varphi^{\prime \prime}+\frac{1-2 \alpha}{y} \varphi^{\prime}-\varphi=0
$$

may be written as $\varphi(y)=y^{\alpha} \psi(y)$, where $\psi$ solves the well known Bessel equation

$$
y^{2} \psi^{\prime \prime}+y \psi^{\prime}-\left(y^{2}+\alpha^{2}\right) \psi=0 .
$$

In addition (2.6) has two linearly independent solutions, $I_{\alpha}, Z_{\alpha}$, which are the modified Bessel functions; their asymptotic behavior is given precisely by

$$
\begin{aligned}
I_{\alpha}(y) \sim & \frac{1}{\Gamma(\alpha+1)}\left(\frac{y}{2}\right)^{\alpha}\left(1+\frac{y^{2}}{4(\alpha+1)}+\frac{y^{4}}{32(\alpha+1)(\alpha+2)}+\ldots\right) . \\
Z_{\alpha}(y) \sim & \frac{\Gamma(\alpha)}{2}\left(\frac{2}{y}\right)^{\alpha}\left(1+\frac{y^{2}}{4(1-\alpha)}+\frac{y^{4}}{32(1-\alpha)(2-\alpha)}+\ldots\right)+ \\
& \quad+\frac{\Gamma(-\alpha)}{2^{\alpha}}\left(\frac{y}{2}\right)^{\alpha}\left(1+\frac{y^{2}}{4(\alpha+1)}+\frac{y^{4}}{32(\alpha+1)(\alpha+2)}+\ldots\right),
\end{aligned}
$$

for $y \rightarrow 0^{+}, \alpha \notin \mathbb{Z}$. And when $y \rightarrow+\infty$,

$$
\begin{aligned}
I_{\alpha}(y) & \sim \frac{1}{\sqrt{2 \pi y}} e^{y}\left(1-\frac{4 \alpha^{2}-1}{8 y}+\frac{\left(4 y^{2}-1\right)\left(4 y^{2}-9\right)}{2 !(8 y)^{2}}+\ldots\right), \\
Z_{\alpha}(y) & \sim \sqrt{\frac{\pi}{2 y}} e^{-y}\left(1-\frac{4 \alpha^{2}-1}{8 y}+\frac{\left(4 y^{2}-1\right)\left(4 y^{2}-9\right)}{2 !(8 y)^{2}}+\ldots\right) .
\end{aligned}
$$

In the sequel, we will use both type of solutions given in Lemma 2.3 above (one is growing exponentially as $y \rightarrow \infty$, one is decaying exponentially to $0)$. Up to a normalization constant chosen in such a way that $\varphi(0)=1$, we set

$$
\varphi_{1}(y):=y^{\alpha} I_{\alpha}(y) \quad \text { and } \quad \varphi_{2}(y):=y^{\alpha} Z_{\alpha}(y)
$$


Remark 2.4. We observe that if $\varphi$ satisfies (2.5), then the function $\bar{\varphi}(y):=$ $\varphi(\mu y)$ is a solution of

$$
\partial_{y}\left(y^{1-2 \alpha} \partial_{y} \bar{\varphi}\right)=y^{1-2 \alpha} \mu^{2} \bar{\varphi} .
$$

Remark 2.5. As said before, $\varphi_{2}$ is the solution of (2.4) and in particular it satisfies

$$
\begin{gathered}
\lim _{y \rightarrow 0} \varphi_{2}(y)=1, \\
-\lim _{y \rightarrow 0} y^{1-2 \alpha} \partial_{y} \varphi_{2}(y)=c_{\alpha},
\end{gathered}
$$

where $c_{\alpha}$ is defined in (2.3).

Moreover, by (2.7) we have

$$
\varphi_{2}(y) \sim y^{\alpha-1 / 2} e^{-y} \text { as } y \rightarrow \infty .
$$

\section{The 1-Dimensional SOlution}

In this section we study existence for solutions of the following problem

$$
\begin{cases}\operatorname{div}\left(y^{1-2 \alpha} \nabla v\right)=0 & \text { in }[0, \lambda] \times(0,+\infty) \\ v=0 & \text { in } \partial[0, \lambda] \times(0,+\infty) \\ -\frac{1}{c_{\alpha}} \lim _{y \rightarrow 0} y^{1-2 \alpha} \partial_{y} v=-F^{\prime}(v) & \text { on }[0, \lambda] \times\{0\}\end{cases}
$$

The energy functional associated to problem (3.1) is given by

$$
E_{0}(v):=\frac{1}{c_{\alpha}} \int_{0}^{+\infty} \int_{0}^{\lambda} \frac{1}{2} y^{1-2 \alpha}|\nabla v(s, y)|^{2} d s d y+\int_{0}^{\lambda} F(v(s, 0)) d s .
$$

We consider the energy functional $E_{0}$ in the class of functions

$$
\begin{gathered}
H_{0, \ell}^{1}\left([0, \lambda] \times(0,+\infty), y^{1-2 \alpha}\right):= \\
\{v:[0, \lambda] \times(0,+\infty) \rightarrow \mathbb{R} \mid v=0 \text { on } \partial[0, \lambda] \times(0,+\infty), \\
\left.y^{1-2 \alpha}\left(|v|^{2}+|\nabla v|^{2}\right) \in L^{1}([0, \lambda] \times(0,+\infty))\right\} .
\end{gathered}
$$

As explained in Section 2 (see $[11,24]$ ), if $v$ solves (3.1) above, than its trace $u$ on $[0, \lambda]$ is a solution of the fractional Dirichlet problem

$$
\left\{\begin{array}{l}
A_{\alpha} u+F^{\prime}(u)=0 \quad \text { in }[0, \lambda] \\
u(0)=u(\lambda)=0 .
\end{array}\right.
$$

In the following lemma, we give a sufficient and necessary condition on the parameter $\lambda$ for the existence of nontrivial solutions to problem (3.1).

Lemma 3.1. Let $\lambda_{*}$ be defined as in (1.6) and assume that $\lambda>\lambda_{*}$ is fixed. Then, there exists a nontrivial positive weak solution of (3.1) which is a minimizer of $E_{0}$ in the space $H_{0, \ell}^{1}\left([0, \lambda] \times(0,+\infty), y^{1-2 \alpha}\right)$. Assume that $\lambda \leq \lambda_{*}$, then there are no positive weak solutions of (3.1) and the trivial solution 0 is the unique minimizer of $E_{0}$. 
Proof. We start by proving the existence of a minimizer for $E_{0}$ in the class $H_{0, \ell}^{1}\left([0, \lambda] \times(0,+\infty), y^{1-2 \alpha}\right)$. Following [14], proof of Theorem 1.6, and [9] proof of Lemma 4.1, we introduce a new potential $\widetilde{F}$ with the following properties:

- $\widetilde{F}=F$ in $[-1,1]$,

- $\widetilde{F}>0$ in $\mathbb{R} \backslash[-1,1]$,

- $\widetilde{F}$ is even,

- $\widetilde{F}$ has linear growth at infinity.

We consider the new energy functional

$$
\widetilde{E}_{0}(v)=\frac{1}{c_{0}} \int_{0}^{+\infty} \int_{0}^{\lambda} \frac{1}{2} y^{1-2 \alpha}|\nabla v(s, y)|^{2} d s d y+\int_{0}^{\lambda} \widetilde{F}(v(s, 0)) d s .
$$

Note that every minimizer $w$ of $\widetilde{E}_{0}$ in $H_{0, \ell}^{1}\left([0, \lambda] \times(0,+\infty), y^{1-2 \alpha}\right)$ such that $-1 \leq w \leq 1$ is also a minimizer of $E_{0}$ in the set

$$
\left\{v \in H_{0, \ell}^{1}\left([0, \lambda] \times(0,+\infty), y^{1-2 \alpha}\right):-1 \leq v \leq 1\right\} .
$$

We show now that $\widetilde{E}_{0}$ admits a minimizer in $H_{0, \ell}^{1}\left([0, \lambda] \times(0,+\infty), y^{1-2 \alpha}\right)$. Indeed, by the properties of $\widetilde{F}$, it follows that $\widetilde{E}_{0}$ is well-defined, bounded below and coercive in $H_{0, \ell}^{1}\left([0, \lambda] \times(0,+\infty), y^{1-2 \alpha}\right)$. Hence, existence of a minimizer for $\widetilde{E}_{0}$ follows using the compactness of the inclusion $H_{0, \ell}^{1}([0, \lambda] \times$ $\left.(0,+\infty), y^{1-2 \alpha}\right) \subset \subset \widetilde{L}^{2}([0, \lambda])$, which was proven in [24], Sect. 4 .

We want now to prove that if $\lambda>\lambda_{*}, 0$ cannot be a minimizer. To this aim, we consider the function

$$
w(s, y):=\sin \left(\frac{\pi}{\lambda} s\right) \varphi_{2}\left(\frac{\pi}{\lambda} y\right)
$$

where $\varphi_{2}$ is defined as in (2.8). By Remark 2.4 and by (2.9) and (2.10) w satisfies the problem

$$
\begin{cases}\operatorname{div}\left(y^{1-2 \alpha} \nabla w\right)=0 & \text { in }[0, \lambda] \times(0,+\infty) \\ w=0 & \text { on } \partial[0, \lambda] \times(0,+\infty) \\ -\frac{1}{c_{\alpha}} \lim _{y \rightarrow 0} y^{1-2 \alpha} \partial_{y} w=\left(\frac{\pi}{\lambda}\right)^{2 \alpha} w & \text { on }[0, \lambda] \times\{y=0\}\end{cases}
$$

We use a small multiple of $w$ as a test function to prove that 0 is not a minimizer when $\lambda>\lambda_{*}$. First of all, we observe that

$$
E_{0}(0)=\lambda F(0) \text {. }
$$

On the other hand, using Taylor expansion for $F$, we have that

$$
\begin{gathered}
E_{0}(\varepsilon w)=\lambda F(0)+\frac{\varepsilon^{2}}{2 c_{\alpha}} \int_{0}^{+\infty} \int_{0}^{\lambda} y^{1-2 \alpha}|\nabla w(s, y)|^{2} d s d y \\
+\frac{\varepsilon^{2}}{2} \int_{0}^{\lambda} F^{\prime \prime}(0) w(s, 0)^{2} d s+\mathcal{O}\left(\varepsilon^{4}\right) .
\end{gathered}
$$


We first observe that

$$
\int_{0}^{\lambda} F^{\prime \prime}(0) w(s, 0)^{2}=F^{\prime \prime}(0) \int_{0}^{\lambda} \sin ^{2}\left(\frac{\pi}{\lambda} s\right) d s=\frac{\lambda}{2} F^{\prime \prime}(0) .
$$

To compute the Dirichlet energy, we use the change of variable $\bar{y}=\frac{\pi}{\lambda} y$ and we integrate by parts in $\bar{y}$ to get

$$
\begin{aligned}
\frac{1}{2 c_{\alpha}} & \int_{0}^{+\infty} \int_{0}^{\lambda} y^{1-2 \alpha}|\nabla w(s, y)|^{2} d s d y= \\
= & \frac{1}{2 c_{\alpha}} \int_{0}^{+\infty} \int_{0}^{\lambda} y^{1-2 \alpha}\left(\frac{\pi}{\lambda}\right)^{2}\left[\cos ^{2}\left(\frac{\pi}{\lambda} s\right) \varphi_{2}^{2}\left(\frac{\pi}{\lambda} y\right)+\sin ^{2}\left(\frac{\pi}{\lambda} s\right) \dot{\varphi}_{2}^{2}\left(\frac{\pi}{\lambda} y\right)\right] d s d y \\
= & \frac{1}{c_{\alpha}} \frac{\lambda}{4}\left(\frac{\pi}{\lambda}\right)^{2 \alpha} \int_{0}^{+\infty} \bar{y}^{1-2 \alpha}\left[\varphi_{2}^{2}(\bar{y})+\dot{\varphi}_{2}^{2}(\bar{y})\right] d \bar{y} \\
= & \frac{1}{c_{\alpha}} \frac{\lambda}{4}\left(\frac{\pi}{\lambda}\right)^{2 \alpha} \int_{0}^{+\infty}\left(\bar{y}^{1-2 \alpha} \varphi_{2}^{2}(\bar{y})-\varphi_{2}(\bar{y}) \partial_{\bar{y}}\left(\bar{y}^{1-2 \alpha} \partial_{\bar{y}} \varphi_{2}(\bar{y})\right)\right) d \bar{y} \\
& -\frac{1}{c_{\alpha}} \frac{\lambda}{4}\left(\frac{\pi}{\lambda}\right)^{2 \alpha} \lim _{\bar{y} \rightarrow 0} \bar{y}^{1-2 \alpha} \varphi_{2}(\bar{y}) \dot{\varphi}_{2}(\bar{y}) \\
= & \frac{\lambda}{4}\left(\frac{\pi}{\lambda}\right)^{2 \alpha},
\end{aligned}
$$

where in the last equality we have used that $\varphi_{2}$ satisfies (2.5) and the asymptotic behaviors (2.9) and (2.10).

Therefore, combining together (3.5) and (3.6), we deduce that

$$
\begin{aligned}
E_{0}(\varepsilon w) & =\lambda F(0)+\frac{\varepsilon^{2}}{4} \lambda\left(\left(\frac{\pi}{\lambda}\right)^{2 \alpha}+F^{\prime \prime}(0)\right)+\mathcal{O}\left(\varepsilon^{4}\right) \\
& <E_{0}(0),
\end{aligned}
$$

for $\varepsilon$ small enough, provided that $\lambda>\lambda_{*}$. This concludes the proof of the first part of the statement, since we get a nontrivial minimizer for $E_{0}$, which can be chosen to be positive by standard arguments.

To prove the nonexistence of positive solutions for $\lambda \leq \lambda_{*}$, we multiply the first equation of (3.1) by $w$ and we integrate by parts, to get

$$
\begin{aligned}
0= & \frac{1}{2 c_{\alpha}} \int_{0}^{+\infty} \int_{0}^{\lambda} \operatorname{div}\left(y^{1-2 \alpha} \nabla v(s, y)\right) w(s, y) d s d y \\
= & -\frac{1}{2 c_{\alpha}} \int_{0}^{+\infty} \int_{0}^{\lambda} y^{1-2 \alpha} \nabla v(s, y) \cdot \nabla w(s, y) d s d y \\
& -\int_{0}^{\lambda} \lim _{y \rightarrow 0} y^{1-2 \alpha} \partial_{y} v(s, y) w(s, 0) d s \\
= & \frac{1}{2 c_{\alpha}} \int_{0}^{\lambda} \lim _{y \rightarrow 0} y^{1-2 \alpha} \partial_{y} w(s, y) v(s, y) d s+\int_{0}^{\lambda} F^{\prime}(v) w(s, 0) d s \\
= & \frac{1}{2} \int_{0}^{\lambda} w(s, 0)\left(F^{\prime}(v)+\left(\frac{\pi}{\lambda}\right)^{2 \alpha} v(s, 0)\right) d s,
\end{aligned}
$$


where in the last two equalities we have used that $w$ satisfies (3.4). This concludes the proof of the lemma, indeed by assumption $F^{\prime}(v) \geq F^{\prime \prime}(0) v$ for any $v \geq 0$, and therefore we must have $v(s, 0) \equiv 0$ when $\lambda \leq \lambda_{*}$, which implies $v \equiv 0$ by uniqueness of solutions to the extended problem (2.2).

For $\lambda>\lambda_{*}$ we will denote by $v_{0}$ the nontrivial minimizer of $E_{0}$, which existence is established in Theorem 3.1. From what we have seen above, we have the inequality:

$$
E_{0}\left(v_{0}\right)<\lambda F(0) .
$$

As a consequence of Lemma 3.1 above, we deduce the following

Corollary 3.2. There exists a nontrivial positive solution of the fractional Dirichlet problem (3.3) on $[0, \lambda]$ if and only if $\lambda>\lambda_{*}$, where $\lambda_{*}$ is defined as in (1.6).

\section{The existence Result For $\lambda>\lambda_{*}$}

This section is devoted to the proof of Theorem 1.1.

In the following we will use cylindrical coordinates $(r, \theta, s, y) \in[0,+\infty) \times$ $\mathbb{S}^{1} \times \mathbb{R} \times \mathbb{R}^{+}$to parametrize $\mathbb{R}^{3} \times \mathbb{R}^{+}$. In order to find a solution of (1.1) which is invariant under the screw motion $\sigma_{\lambda}^{\beta}$, we will look for a solution $v$ of the extended problem (1.7) which is invariant under the transformation, that for simplicity of notation we still denote by $\sigma_{\lambda}^{\beta}$, acting on $\mathbb{R}^{3} \times \mathbb{R}^{+}$in the natural way

$$
\sigma_{\lambda}^{\beta}(z, s, y)=\left(e^{i \beta} z, s+\frac{\lambda}{\pi} \beta, y\right) .
$$

More precisely, we require that

$$
v(r, \theta, s, y)=v\left(r, \theta+\beta, s+\frac{\lambda}{\pi} \beta, y\right) \quad \text { for every } \beta \in \mathbb{R},
$$

which implies that

$$
\begin{gathered}
v(r, \theta, s, y)=v(r, \theta, s+2 \lambda, y) \quad \text { and } \\
v(r, \theta, s, y)=v\left(r, 0, s-\frac{\lambda}{\pi} \theta, y\right) .
\end{gathered}
$$

Moreover, we assume that

$$
v(r, \theta,-s, y)=-v(r, \theta, s, y) .
$$

Using these invariances, it is clear that in order to construct the solution $v$, it is enough to construct it for $\theta=0$, that is we just need to know

$$
V(r, s, y)=v(r, 0, s, y) \quad \text { for }(r, s, y) \in[0,+\infty) \times[0, \lambda] \times \mathbb{R}^{+} .
$$

Indeed, once we have $V(r, s, y)$ defined on $[0,+\infty) \times[0, \lambda] \times \mathbb{R}^{+}$it is enough to reflect it oddly with respect to $\{s=0\}$ and $\{s=\lambda\}$; in this way we obtain a function $V(r, s, \lambda)$ defined on all $[0,+\infty) \times \mathbb{R} \times \mathbb{R}^{+}$. Finally we use (4.1) to define $v$ in terms of $V$. Of course, by construction, we have that if $V$ is positive in $(0,+\infty) \times(0, \lambda) \times \mathbb{R}^{+}$and vanishes on $\partial([0,+\infty) \times[0, \lambda]) \times \mathbb{R}^{+}$, 
then the zero level set of $v$ is $H_{\lambda} \times \mathbb{R}^{+}$, and therefore the zero level set of its trace $u$ on $\{y=0\}$, that is a solution of (1.1), is exactly the helicoid $H_{\lambda}$.

We need now to write problem (1.7) and the energy (1.8) in the cylindrical coordinates introduced above for functions invariant under screw motion. Problem (1.7) becomes

$$
\begin{cases}V_{r r}+\frac{1}{r} V_{r}+\left(1+\frac{\lambda^{2}}{\pi^{2} r^{2}}\right) V_{s s}+V_{y y}+\frac{1-2 \alpha}{y} V_{y}=0 & \text { in }[0,+\infty) \times[0, \lambda] \times \mathbb{R}^{+}, \\ V=0 & \text { on }\{s=0\} \cup\{s=\lambda\} \\ -\frac{1}{c_{\alpha}} \lim _{y \rightarrow 0} y^{1-2 \alpha} \partial_{y} V=-F^{\prime}(V) & \text { on }\{y=0\}\end{cases}
$$

In what follows we will use the following notations:

$$
S_{R}:=[0, R] \times[0, \lambda] \quad \text { and } \quad C_{R, L}:=S_{R} \times[0, L] .
$$

We define the following subsets of $\partial C_{R, L}$ :

$$
\begin{aligned}
\partial^{+} C_{R, L} & :=\overline{\partial C_{R, L} \cap\{y>0\}}, \\
\partial^{0} C_{R, L} & :=\partial C_{R, L} \backslash \partial^{+} C_{R, L} .
\end{aligned}
$$

The energy associated to problem (4.2) in the cylinder $C_{R, L}$ is given by:

$$
\begin{aligned}
E\left(V, C_{R, L}\right)=\frac{1}{2 c_{\alpha}} & \iint_{C_{R, L}} y^{1-2 \alpha}\left(\left|V_{r}\right|^{2}+\left(1+\frac{\lambda^{2}}{\pi^{2} r^{2}}\right)\left|V_{s}\right|^{2}+\left|V_{y}\right|^{2}\right) r d r d s d y \\
& +\int_{\partial^{0} C_{R, L}} F(V) r d r d s .
\end{aligned}
$$

Let $H^{1}\left(C_{R, L}, y^{1-2 \alpha}\right)$ denote the weighted Sobolev space

$$
H^{1}\left(C_{R, L}, y^{1-2 \alpha}\right)=\left\{v: C_{R, L} \rightarrow \mathbb{R} \mid y^{1-2 \alpha}\left(v^{2}+|\nabla v|^{2}\right) \in L_{r}^{1}\left(C_{R, L}\right)\right\},
$$

where $L_{r}^{1}$ denotes the space $L^{1}\left(C_{R, L}\right)$ with respect to the measure $r d r d s d y$, and let $\tilde{H}_{0}^{1}\left(C_{R, L}, y^{1-2 \alpha}\right)$ be the space

$$
\tilde{H}_{0}^{1}\left(C_{R, L}, y^{1-2 \alpha}\right)=\left\{v \in H^{1}\left(C_{R, L}, y^{1-2 \alpha}\right)|| v \mid \leq 1, v \equiv 0 \text { on } \partial^{+} C_{R, L}\right\} \text {. }
$$

We recall that (see the proof of Lemma 4.1 in [9]), the inclusion

$$
\tilde{H}_{0}^{1}\left(C_{R, L}, y^{1-2 \alpha}\right) \subset \subset L^{2}\left(\partial^{0} C_{R, L}\right)
$$

is compact.

We are now ready to give the prove of Theorem 1.1.

Proof of Theorem 1.1. Similarly to the proof of Lemma 3.1, we introduce the functional $\widetilde{E}\left(\cdot, C_{R, L}\right)$ obtained just replacing the potential $F$ with the function $\widetilde{F}$ (that is the same of the one in the proof of Lemma 3.1). We observe as before that every minimizer $W$ of $\widetilde{E}$ in $\tilde{H}_{0}^{1}\left(C_{R, L}, y^{1-2 \alpha}\right)$ such that $|W| \leq 1$, is also a minimizer for $E$ in the class $\left\{V \in H_{0}^{1}\left(C_{R, L}, y^{1-2 \alpha}\right):|V| \leq 1\right\}$. By a standard variational argument and using compactness of the inclusion (4.3), taking a minimizing sequence $\left\{V_{R, L}^{k}\right\} \in \tilde{H}_{0}^{1}\left(C_{R, L}, y^{1-2 \alpha}\right)$ and a subsequence convergent in $L^{2}\left(\partial^{0} C_{R, L}\right)$, we conclude that $\widetilde{E}\left(\cdot, C_{R, L}\right)$ admits an 
absolute minimizer $V_{R, L}$ in $\tilde{H}_{0}^{1}\left(C_{R, L}, y^{1-2 \alpha}\right)$. Note moreover that, without loss of generality, we may assume that $0 \leq V_{R, L} \leq 1$ because, if not, we can replace the minimizing sequence $V_{R, L}^{k}$ with the sequence $\min \left\{\left|V_{R, L}^{k}\right|, 1\right\}$. Indeed, it is also minimizing because $\widetilde{F}$ is even and $\widetilde{F} \geq \widetilde{F}(1)$.

It is easy to check that $V_{R, L}$ is a solution of (4.2) in $C_{R, L}$ (with 0-Dirichlet boundary condition on $\partial^{+} C_{R, L}$ ). Arguing as in the proof of Theorem 1.3 in [14], one can prove that $V_{R, L}$ extends to a solution of

$$
\begin{cases}\operatorname{div}\left(y^{1-2 \alpha} \nabla v\right)=0 & \text { in } C_{R, L} \\ v \equiv 0 & \text { on } \partial^{+} C_{R, L} \\ -\frac{1}{c_{\alpha}} \lim _{y \rightarrow 0} y^{1-2 \alpha} \partial_{y} v=-F^{\prime}(v) & \text { on } \partial^{0} C_{R, L},\end{cases}
$$

where, for simplicity, we keep the notation $C_{R, L}$ to denote the corresponding cylinder in $\mathbb{R}_{+}^{4}$, that is

$$
C_{R, L}=\left\{(x, y)=(r, \theta, s, y) \in \mathbb{R}_{+}^{4}: 0<r<R, 0<y<L\right\},
$$

and the subsets of its boundary

$$
\partial^{+} C_{R, L}=\overline{\partial C_{R, L} \cap \mathbb{R}_{+}^{4}}, \quad \partial^{0} C_{R, L}:=\partial C_{R, L} \backslash \partial^{+} C_{R, L} .
$$

Some care is needed to show that it is a solution close to $\{r=0\}$, and we refer to [14] for details. We now wish to pass to the limit in $R$ and $L$, and obtain a solution in all of $[0, \infty) \times[0, \lambda] \times \mathbb{R}^{+}$. Let $S>0, L^{\prime}>0$ and consider the family $\left\{V_{R, L}\right\}$ of solutions in $[0, S+2] \times[0, \lambda] \times\left[0, L^{\prime}+2\right]$, with $R>S+2$ and $L>L^{\prime}+2$. Since $\left|V_{R, L}\right| \leq 1$, regularity results proven in Proposition 4.6 of [8] , give a bound in $C^{\gamma}\left([0, S] \times[0, \lambda] \times\left[0, L^{\prime}\right]\right)$ for $V_{R, L}$, for some $\gamma \in(0,1)$, uniform with respect to $R$ and $L$.

Choose now $L=R^{b}$, with $1 / 2<b<1$ (this choice will be used later to prove that the solution that we construct is not identically zero). By the Ascoli-Arzelà Theorem and a standard diagonal argument, there is a sequence $V_{R_{j}, R_{j}^{b}}$ that converges in $C^{\gamma}(K)$ for each compact $K \subset[0, \infty) \times$ $[0, \lambda] \times[0, \infty)$ to a function $V \in C^{\gamma}([0, \infty) \times[0, \lambda] \times[0, \infty))$, which is a solution of (4.2). By construction we also have $|V| \leq 1$.

It only remains to prove that $V$ is positive.

We start by proving that $V$ is not identically zero. In order to do that, following the argument in [14], we establish an energy estimate for $V$ using a comparison argument, based on the minimality of $V_{R, R^{b}}$ in the set $C_{R, R^{b}}$.

Suppose by contradiction that $\lambda>\lambda_{*}$ and that $V \equiv 0$. Then, given $R>0$, the minimizing sequence $V_{R, R^{b}}$ converges uniformly to 0 on $C_{R, R^{b}}$ when $R \rightarrow+\infty$. The energy of 0 in $C_{R, R^{b}}$ is clearly

$$
E\left(0, C_{R, R^{b}}\right)=\frac{\lambda}{2} F(0) R^{2} .
$$

Therefore, for any function $W$ vanishing on $\partial^{+} C_{R, R^{b}}$, we have that

$$
E\left(W, C_{R, R^{b}}\right) \geq E\left(0, C_{R, R^{b}}\right)=\frac{\lambda}{2} F(0) R^{2} .
$$


We build now a suitable competitor $W$ and we arrive to a contradiction with (4.4). First we define two smooth cut-off functions $\eta$ and $\xi$ as follows:

$$
\eta(r):= \begin{cases}0 & \text { for } r \in[0,1 / 2] \cup[R-1 / 2, R] \\ 1 & \text { for } r \in[1, R-1]\end{cases}
$$

and

We set

$$
\xi(y)= \begin{cases}1 & \text { if } 0<y \leq R^{b}-R^{a} \\ \frac{\log R^{b}-\log y}{\log R^{b}-\log \left(R^{b}-R^{a}\right)} & \text { if } R^{b}-R^{a}<y \leq R^{b} .\end{cases}
$$

$$
W(r, s, y):=\eta(r) \xi(y) v_{0}(s, y)
$$

where $v_{0}$ is the minimizer of $E_{0}$ (see (3.2)), whose existence is established in Lemma 3.1 and which satisfies (3.7).

We compute now the energy of $W$. First, we observe that the potential energy is estimated by

$$
\int_{0}^{\lambda} \int_{0}^{R} F(W(r, s, 0)) r d r d s \leq \frac{R^{2}}{2} \int_{0}^{\lambda} F\left(v_{0}(s, 0)\right) d s+\mathcal{O}(R) .
$$

We estimate now the Dirichlet energy. Since $|\eta|,|\xi|,\left|v_{0}\right| \leq 1$, we have that:

$$
\begin{aligned}
& \frac{1}{2 c_{\alpha}} \iint_{C_{R, R^{b}}} y^{1-2 \alpha}|\nabla W|^{2} d x d y \\
& \quad \leq \frac{1}{2 c_{\alpha}} \iint_{C_{R, R^{b}}} y^{1-2 \alpha}\left(|\nabla \eta|^{2}+\left|\nabla v_{0}\right|^{2}+|\dot{\xi}|^{2}\right) d x d y .
\end{aligned}
$$

This, together with (4.5), implies

$E\left(W, C_{R, R^{b}}\right) \leq \frac{R^{2}}{2} E_{0}\left(v_{0}\right)+\frac{1}{2 c_{\alpha}} \iint_{C_{R, R^{b}}} y^{1-2 \alpha}\left(|\nabla \eta|^{2}+|\dot{\xi}|^{2}\right) d x d y+\mathcal{O}(R)$.

We estimate now the second term on the right-hand side above. In the following, $C_{\alpha}$ will denote positive, possibly different, constants depending only on $\alpha$. Using the definition of $\eta$, we have that

$$
\iint_{C_{R, R^{b}}} y^{1-2 \alpha}|\nabla \eta|^{2} d x d y \leq C_{\alpha} \lambda R \int_{0}^{R^{b}} y^{1-2 \alpha} d y=C_{\alpha} \lambda R^{1+2 b(1-\alpha)} .
$$

On the other hand, by the definition of $\xi$ and using polar coordinates, we have

$$
\begin{aligned}
& \int_{0}^{R^{b}} \int_{0}^{\lambda} \int_{0}^{R} y^{1-2 \alpha}|\dot{\xi}(y)|^{2} r d r d s d y \leq C_{\alpha} \lambda R^{2} \frac{1}{\left(\log \frac{R^{b}}{R^{b}-R^{a}}\right)^{2}} \int_{R^{b}-R^{a}}^{R^{b}} \frac{y^{1-2 \alpha}}{y^{2}} d y \\
& \leq C_{\alpha} \lambda R^{2} \frac{1}{-\log \left(1-R^{a-b}\right)^{2}}\left[\frac{1}{R^{2 \alpha b}-R^{2 \alpha a}}-\frac{1}{R^{2 \alpha b}}\right] \\
& \leq C_{\alpha} \lambda R^{2} \cdot R^{2(b-a)} \cdot R^{-2 \alpha b}=C_{\alpha} \lambda R^{2+2 b(1-\alpha)-2 a}
\end{aligned}
$$


Therefore, plugging (4.5), (4.7) and (4.8) into (4.6), we deduce

$$
E\left(W, C_{R, R^{b}}\right) \leq C_{\alpha} \lambda R^{1+2 b(1-\alpha)}+C_{\alpha} \lambda R^{2+2 b(1-\alpha)-2 a}+\frac{R^{2}}{2} E_{0}\left(v_{0}\right)+\mathcal{O}(R) .
$$

Now we choose $1 / 2<a<b<\frac{1}{2(1-\alpha)}$. With this choice of $b$ and $a$, there exists $\varepsilon=\varepsilon(b)$ such that

$$
E\left(W, C_{R, R^{b}}\right) \leq C_{\alpha} \lambda R^{2-\varepsilon}+\frac{R^{2}}{2} E_{0}\left(v_{0}\right)+\mathcal{O}\left(R^{2-\varepsilon}\right) .
$$

This, together with (4.4), implies

$$
\frac{1}{2} F(0) R^{2} \leq \frac{R^{2}}{2} E_{0}\left(v_{0}\right)+\mathcal{O}(R) .
$$

This gives a contradiction, since for $\lambda>\lambda_{*}$ we have that

$$
E_{0}\left(v_{0}\right)<\lambda F(0),
$$

which was established in (3.7).

This implies that $V$ is not identically zero. Since by construction $V \geq 0$, we conclude that $V$ is strictly positive using the strong maximum principle.

\section{The nOneXistence Result}

In this section we give the proof of our nonexistence result Theorem 1.2.

In order to do this, we need to establish exponential decay in the $y$ variable of a bounded solution $v$ of (1.7) which vanishes on $H_{\lambda} \times \mathbb{R}^{+}$and is invariant under screw motion. We stress that in general exponential decay in the $y$ variable for bounded solutions in all the half-space of problem (1.7) is not true, as one can see in the particular case of the one-dimensional Peierls-Nabarro problem:

$$
\begin{cases}\Delta v=0 & \text { in } \mathbb{R}_{+}^{2} \\ -\partial_{y} v(x, 0)=\frac{1}{\pi} \sin (\pi u) & \text { on } \mathbb{R},\end{cases}
$$

for which an explicit solution is given by $v(x, y)=\frac{2}{\pi} \arctan \left(\frac{x}{1+y}\right)$ (see [10] and references therein). On the other hand, as shown in Lemmas 2.2 and 2.3, the solution of problem (2.2) in a cylinder $\mathcal{C}_{\Omega}=\Omega \times \mathbb{R}^{+}$, with $\Omega$ bounded, with 0 -Dirichlet condition on the lateral boundary $\partial \Omega \times \mathbb{R}^{+}$decays exponentially in $y$. In our situation, we are able to prove exponential decay, thanks to the symmetry of the problem and to the invariances of the solution $v$.

Proposition 5.1. Suppose that $v$ is a bounded solution of

$$
\begin{cases}\operatorname{div}\left(y^{1-2 \alpha} \nabla v\right)=0 & \text { in } \mathbb{R}_{+}^{4} \\ -\frac{1}{c_{\alpha}} \lim _{y \rightarrow 0} y^{1-2 \alpha} \partial_{y} v(x, y)=-F^{\prime}(v), & \end{cases}
$$

which vanishes on $H_{\lambda} \times \mathbb{R}^{+}$and is invariant under screw motion. 
Then, there exist a positive constant $K$ such that

$$
|v(x, y)| \leq K \varphi_{2}(y) \sim y^{\alpha-\frac{1}{2}} e^{-y} \quad \text { as } y \rightarrow \infty .
$$

Proof. Using polar coordinates and the invariance of $v$, as done in Section 4 , we have that the function $V(r, s, y)=v(r, 0, s, y)$ satisfies:

$$
\begin{cases}V_{r r}+\frac{1}{r} V_{r}+\left(1+\frac{\lambda^{2}}{\pi^{2} r^{2}}\right) V_{s s}+V_{y y}+\frac{1-2 \alpha}{y} V_{y}=0 & \text { in }[0,+\infty) \times[0, \lambda] \times \mathbb{R}^{+}, \\ V=0 & \text { on } s \in\{0, \lambda\}, \\ -\frac{1}{c_{\alpha}} \lim _{y \rightarrow 0} y^{1-2 \alpha} \partial_{y} v(x, y)=-F^{\prime}(v) . & \end{cases}
$$

We define now the following function, which provides an upper barrier for $V$. For $C>e^{2}, K$ large enough to be chosen later, and $\varepsilon>0$, we set

$$
w^{\varepsilon}(r, s, y):=K \sin \left(\frac{\pi}{\lambda} s\right) \cdot\left[\varphi_{2}\left(\frac{\pi}{\lambda} y\right)+\varepsilon \varphi_{1}\left(\frac{\pi}{\lambda} y\right)+\varepsilon\left(e^{\frac{1}{2} \frac{\pi}{\lambda} r}+C e^{-\frac{1}{2} \frac{\pi}{\lambda} r}\right)\right],
$$

where $\varphi_{1}$ and $\varphi_{2}$ were defined in (2.8). To conclude the proof, it is enough to prove that, for any $\varepsilon>0$ (sufficiently small),

$$
V \leq w^{\varepsilon}
$$

Then, the conclusion follows by sending $\varepsilon \rightarrow 0$.

We start by showing that $w_{\varepsilon}$ satisfies:

$$
w_{r r}^{\varepsilon}+\frac{1}{r} w_{r}^{\varepsilon}+\left(1+\frac{\lambda^{2}}{\pi^{2} r^{2}}\right) w_{s s}^{\varepsilon}+w_{y y}^{\varepsilon}+\frac{1-2 \alpha}{y} w_{y}^{\varepsilon} \leq 0
$$

By a direct computation, we have that:

$$
\begin{aligned}
\frac{1}{K}\left[w_{r r}^{\varepsilon}\right. & \left.+\frac{1}{r} w_{r}^{\varepsilon}+\left(1+\frac{\lambda^{2}}{\pi^{2} r^{2}}\right) w_{s s}^{\varepsilon}+w_{y y}^{\varepsilon}+\frac{1-2 \alpha}{y} w_{y}^{\varepsilon}\right] \\
= & -\frac{3}{4} \varepsilon\left(\frac{\pi}{\lambda}\right)^{2} \sin \left(\frac{\pi}{\lambda} s\right)\left(e^{\frac{1}{2} \frac{\pi}{\lambda} r}+C e^{-\frac{1}{2} \frac{\pi}{\lambda} r}\right) \\
& +\frac{1}{2 r} \varepsilon \frac{\pi}{\lambda} \sin \left(\frac{\pi}{\lambda} s\right)\left(e^{\frac{1}{2} \frac{\pi}{\lambda} r}-C e^{-\frac{1}{2} \frac{\pi}{\lambda} r}\right)-\frac{1}{r^{2}} w^{\varepsilon} \\
\leq & -\frac{3}{4} \varepsilon\left(\frac{\pi}{\lambda}\right)^{2} \sin \left(\frac{\pi}{\lambda} s\right)\left(e^{\frac{1}{2} \frac{\pi}{\lambda} r}+C e^{-\frac{1}{2} \frac{\pi}{\lambda} r}\right) \\
& +\frac{1}{2 r} \varepsilon \frac{\pi}{\lambda} \sin \left(\frac{\pi}{\lambda} s\right)\left(e^{\frac{1}{2} \frac{\pi}{\lambda} r}-C e^{-\frac{1}{2} \frac{\pi}{\lambda} r}\right)=A_{1}+A_{2} .
\end{aligned}
$$

We study now separately the cases $r \geq \frac{\lambda}{\pi}$ and $r<\frac{\lambda}{\pi}$.

If $r \geq \frac{\lambda}{\pi}$, the last term in (5.4) is bounded above by

$$
\begin{aligned}
A_{2} & \leq \frac{1}{2} \varepsilon\left(\frac{\pi}{\lambda}\right)^{2} \sin \left(\frac{\pi}{\lambda} s\right)\left(e^{\frac{1}{2} \frac{\pi}{\lambda} r}-C e^{-\frac{1}{2} \frac{\pi}{\lambda} r}\right) \\
& \leq \frac{1}{2} \varepsilon\left(\frac{\pi}{\lambda}\right)^{2} \sin \left(\frac{\pi}{\lambda} s\right)\left(e^{\frac{1}{2} \frac{\pi}{\lambda} r}+C e^{-\frac{1}{2} \frac{\pi}{\lambda} r}\right) .
\end{aligned}
$$

This, combined with (5.4) implies (5.3) when $r \geq \frac{\lambda}{\pi}$.

When $r<\frac{\lambda}{\pi}$, we immediately deduce that

$$
e^{\frac{1}{2} \frac{\pi}{\lambda} r}-C e^{-\frac{1}{2} \frac{\pi}{\lambda} r} \leq 0,
$$


since we have chosen $C>e^{2}$ and therefore (5.3) holds. It remains to prove that $w^{\varepsilon} \geq V$ on $\partial\left([0,+\infty) \times[0, \lambda] \times \mathbb{R}^{+}\right)$. On the set $\{s=0\} \cup\{s=\lambda\}$, this is easy, since by definition $w^{\varepsilon}=0=V$ while on $\{r=0\}$ we have $w^{\varepsilon} \geq 0=V$. When $y \rightarrow \infty$ and $r \rightarrow \infty$, it is also true. Indeed $V$ is bounded and, for any fixed $\varepsilon, w^{\varepsilon}$ can be made arbitrarily large for $y$ and $r$ sufficiently large. To conclude we just have to prove that $w^{\varepsilon} \geq V$ on $\{y=0\}$. On this part of the boundary, by definition of $w^{\varepsilon}$, we have that for any $\varepsilon>0$

$$
w^{\varepsilon}(r, s, 0) \geq K \sin \left(\frac{\pi}{\lambda} s\right),
$$

since $\varphi_{1}(0)=\varphi_{2}(0)=1$. By Proposition 4.6 in [8], we know that $v$ has bounded $x$-gradient, in particular $v_{s}=V_{s}$ is bounded. Therefore, since $V=0$ on $\{s=0\} \cup\{s=\lambda\}$, we deduce that, there exists a constant $\tilde{C}$ such that

$$
|V(r, s, 0)| \leq \tilde{C} \min \{s, \lambda-s\} .
$$

To conclude, we observe that it is possible to choose $K$ sufficiently large independently of $\varepsilon$, so that

$$
w^{\varepsilon}(r, s, 0) \geq K \sin \left(\frac{\pi}{\lambda} s\right) \geq \tilde{C} \min \{s, \lambda-s\} \geq|V(r, s, 0)| .
$$

We remark that $K$ can be chosen independently of $\varepsilon$ since it only needs to satisfy the second inequality in (5.5) above, in which $\epsilon$ does not appear anymore. We have proven that $w^{\varepsilon}$ is an upper barrier for $V$ and therefore (5.2) holds. This concludes the proof of the proposition.

We can now prove our non-existence result.

Proof of Theorem 1.2. We start by observing that, by uniqueness of solutions to problem

$$
\left\{\begin{array}{l}
\operatorname{div}\left(y^{1-2 \alpha} \nabla v\right)=0 \quad \text { in } \mathbb{R}_{+}^{n+1} \\
v(x, 0)=u(x)
\end{array}\right.
$$

and since the operator $\operatorname{div}\left(y^{1-2 \alpha} \nabla\right)$ is invariant under the screw motion $\sigma_{\lambda}^{\beta}$, in order to prove Theorem 1.2, it is enough to prove the corresponding non-existence result for the extended problem (1.7). We write $V(r, s, y)=$ $v(r, 0, s, y)$ and we consider problem (4.2) written in cylindrical coordinates. Let $\eta$ be a cut-off function only depending on $r$ such that

$$
\eta(r)=\left\{\begin{array}{ll}
1 & r \leq R \\
0 & r \geq 2 R
\end{array} \text { and } \quad|\nabla \eta| \leq \frac{C}{R} .\right.
$$

We multiply (4.2) by $V \eta^{2}$ and we integrate by parts, to obtain

$$
\begin{aligned}
& \int_{0}^{+\infty} \int y^{1-2 \alpha}\left(\left|V_{r}\right|^{2}+\left(1+\frac{\lambda^{2}}{\pi^{2} r^{2}}\right)\left|V_{s}\right|^{2}+\left|V_{y}\right|^{2}\right) \eta^{2} r d r d s d y \\
& \quad+\int F^{\prime}(V) V \eta^{2} r d r d s=2 \int_{0}^{+\infty} \int y^{1-2 \alpha} V V_{r} \eta \eta_{r} r d r d s d y,(5.6)
\end{aligned}
$$


where the domain of integration is $[0, \infty) \times[0, \lambda]$ where it is not explicitly written. Now we use the assumption $F^{\prime}(t) t \geq F^{\prime \prime}(0) t^{2}$ for any $t \in \mathbb{R}$, to get

$$
\begin{aligned}
& \int_{0}^{\infty} \int_{0}^{\lambda} y^{1-2 \alpha}\left(\left|V_{s}\right|^{2}+\left|V_{y}\right|^{2}\right) d s d y+\int_{0}^{\lambda} F^{\prime}(V) V d s \\
& \quad \geq \int_{0}^{\infty} \int_{0}^{\lambda} y^{1-2 \alpha}\left(\left|V_{s}\right|^{2}+\left|V_{y}\right|^{2}\right) d s d y+\int_{0}^{\lambda} F^{\prime \prime}(0) V^{2} d s .
\end{aligned}
$$

We want now to use the stability of $V$ to prove that the quantity above is nonnegative. Since $V$ is not compactly supported in $y$, in order to do that, we use a truncation argument in the $y$-variable. More precisely, given $L>1$, let $\psi$ be a cut-off function depending only on $y$ such that $\psi(y)=1$ for $0<y<L-1$ and $\psi(y)=0$ for $y>L$. We set $V^{L}:=V \psi$, so that $V_{s}^{L}=V_{s} \psi$ and $V_{y}^{L}=V_{y} \psi+V \psi^{\prime}$.

Using $V^{L}$ as a test function in the stability inequality and the fact that 0 is the unique absolute minimizer for the one-dimensional problem when $\lambda \leq \lambda_{*}$, we have

$$
\begin{aligned}
0 \leq & \int_{0}^{\infty} y^{1-2 \alpha}\left(\left|V_{s}^{L}\right|^{2}+\left|V_{y}^{L}\right|^{2}\right) d s d y+\int_{0}^{\lambda} F^{\prime \prime}(0)\left(V^{L}\right)^{2} d s \\
\leq & \int_{0}^{L} y^{1-2 \alpha}\left(\left|V_{s}\right|^{2}+\left|V_{y}\right|^{2}\right) \psi^{2} d s d y+\int_{L-1}^{L} \int_{0}^{\lambda}\left(\psi^{\prime 2} V^{2}+2 \psi \psi^{\prime} V V_{y}\right) \\
& +\int_{0}^{\lambda} F^{\prime \prime}(0)\left(V^{L}\right)^{2} d s .
\end{aligned}
$$

Using now that $\psi \leq 1, \psi^{\prime}$ and $V_{y}$ are bounded for $y \in(L-1, L)$ and the exponential decay for $V$ established in Proposition (5.1), passing to the limit as $L \rightarrow \infty$ we get

$$
\int_{0}^{\infty} \int_{0}^{\lambda} y^{1-2 \alpha}\left(\left|V_{s}\right|^{2}+\left|V_{y}\right|^{2}\right) d s d y+\int_{0}^{\lambda} F^{\prime \prime}(0) V^{2} d s \geq 0 .
$$

Therefore, from (5.6) we deduce

$$
\int_{0}^{\infty} \int y^{1-2 \alpha}\left|V_{r}\right|^{2} \eta^{2} r d r d s d y \leq 2 \int_{0}^{\infty} \int y^{1-2 \alpha} V V_{r} \eta \eta_{r} r d r d s d y
$$

Using Cauchy-Schwarz inequality on the right-hand side we have

$$
\begin{gathered}
\int_{0}^{\infty} \int y^{1-2 \alpha}\left|V_{r}\right|^{2} \eta^{2} r d r d s d y \leq\left(\int_{0}^{\infty} \int_{r \in[R, 2 R]} y^{1-2 \alpha}\left|V_{r}\right|^{2} \eta^{2} r d r d s d y\right)^{\frac{1}{2}} . \\
\cdot\left(\int_{0}^{\infty} \int_{r \in[R, 2 R]} y^{1-2 \alpha}|V|^{2}\left|\eta_{r}\right|^{2} r d r d s d y\right)^{\frac{1}{2}}
\end{gathered}
$$

Now, using that $|\nabla \eta| \leq \frac{C}{R}$ and the exponential decay (5.1) of $V$ in the variable $y$, we deduce that the second integral on the right-hand side is 
bounded, which implies that the integral

$$
\int_{0}^{\infty} \int_{r \in[R, 2 R]} y^{1-2 \alpha}\left|V_{r}\right|^{2} \eta^{2} r d r d s d y
$$

is bounded independently of $R$. Letting $R$ tend to infinity, we conclude that

$$
\int_{0}^{\infty} \int y^{1-2 \alpha}\left|V_{r}\right|^{2} r d r d s d y \leq C
$$

In particular, there exists a sequence $R_{i} \rightarrow \infty$ for which

$$
\lim _{i \rightarrow \infty} \int_{0}^{\infty} \int_{r \in\left[R_{i}, 2 R_{i}\right]} y^{1-2 \alpha}\left|V_{r}\right|^{2} \eta^{2} r d r d s d y=0
$$

This, together with (5.7), implies that

$$
\int_{0}^{\infty} \int y^{1-2 \alpha}\left|V_{r}\right|^{2} r d r d s d y \leq 0
$$

which concludes the proof.

\section{Proof of Theorem 1.3}

In this last section we prove that helicoids have vanishing nonlocal mean curvature.

Proof of Theorem 1.3. We have to show that

$$
\mathcal{H}_{H_{\lambda}}^{2 \alpha}\left(x_{0}\right)=0
$$

for all $x_{0} \in H_{\lambda}$. We can assume for this calculation that $x_{0}=\left(t_{0}, 0,0\right)=$ $\left(t_{0} e^{i \cdot 0}, 0\right)$ where $t_{0}>0$.

We can write

$$
\mathbb{R}^{3} \backslash H_{\lambda}=E_{+} \cup E_{-}
$$

where

$$
\begin{aligned}
& E_{+}=\left\{\left(t e^{i \theta}, \frac{\lambda}{\pi}(\theta+z)\right) \mid t>0, \theta \in \mathbb{R}, z \in(0, \pi)\right\} \\
& E_{-}=\left\{\left(t e^{i \theta}, \frac{\lambda}{\pi}(\theta+z)\right) \mid t<0, \theta \in \mathbb{R}, z \in(0, \pi)\right\}
\end{aligned}
$$

are the two connected components of $\mathbb{R}^{3} \backslash H_{\lambda}$. We observe that the double helicoid $H_{\lambda}$ is the boundary of $E_{+}$and that $E_{+}^{C}=E_{-}$(up to a measure zero set). Then, the nonlocal mean curvature of $H_{\lambda}=\partial E_{+}$is given by

$$
\mathcal{H}_{H_{\lambda}}^{2 \alpha}\left(x_{0}\right)=\int_{\mathbb{R}^{3}} \frac{\chi_{E_{+}}(x)-\chi_{E_{-}}(x)}{\left|x-x_{0}\right|^{3+2 \alpha}} d x .
$$

Consider the transformation

$$
f\left(t e^{i \theta}, \frac{\lambda}{\pi}(\theta+z)\right)=\left(t e^{-i \theta},-\frac{\lambda}{\pi}(\theta+z)\right)
$$


defined for $\left(t e^{i \theta}, \frac{\lambda}{\pi}(\theta+z)\right) \in E_{+}$. Let us verify that $\left(t e^{-i \theta},-\frac{\lambda}{\pi}(\theta+z)\right) \in E_{-}$ if $\left(t e^{i \theta}, \frac{\lambda}{\pi}(\theta+z)\right) \in E_{+}$. Indeed

$$
\left(t e^{-i \theta},-\frac{\lambda}{\pi}(\theta+z)\right)=\left(-t e^{i(-\pi-\theta)}, \frac{\lambda}{\pi}(-\pi-\theta+(\pi-z))\right) \in E_{-}
$$

if $t>0$ and $z \in(0, \pi)$. Moreover $f$ is a bijection from $E_{+}$onto $E_{-}$, which preserves volume. Moreover, writing $x=\left(t e^{i \theta}, \frac{\lambda}{\pi}(\theta+z)\right) \in E_{+}, x^{*}=f(x)=$ $\left(t e^{-i \theta},-\frac{\lambda}{\pi}(\theta+z)\right) \in E_{-}$and $x_{0}=\left(t_{0}, 0,0\right)$, we have

$$
\begin{aligned}
\left|x-x_{0}\right|^{2} & =\left(t \cos (\theta)-t_{0}\right)^{2}+t^{2} \sin ^{2}(\theta)+\frac{\lambda^{2}}{\pi^{2}}(\theta+z)^{2} \\
& =\left|x^{*}-x_{0}\right|^{2}
\end{aligned}
$$

Therefore, changing $x$ to $x^{*}=f(x)$ in the second integral below

$$
\begin{aligned}
\mathcal{H}_{H_{\lambda}}^{2 \alpha}\left(x_{0}\right) & =\lim _{r \rightarrow 0} \int_{\left\{\left|x-x_{0}\right| \geq r\right\}} \frac{\chi_{E_{+}}(x)-\chi_{E_{-}}(x)}{\left|x-x_{0}\right|^{3+2 \alpha}} d x \\
& =\lim _{r \rightarrow 0}\left(\int_{\left\{\left|x-x_{0}\right| \geq r\right\} \cap E_{+}} \frac{1}{\left|x-x_{0}\right|^{3+2 \alpha}} d x-\int_{\left\{\left|x-x_{0}\right| \geq r\right\} \cap E_{-}} \frac{1}{\left|x-x_{0}\right|^{3+2 \alpha}} d x\right) \\
& =\lim _{r \rightarrow 0}\left(\int_{\left\{\left|x-x_{0}\right| \geq r\right\} \cap E_{+}} \frac{1}{\left|x-x_{0}\right|^{3+2 \alpha}} d x-\int_{\left\{\left|x-x_{0}\right| \geq r\right\} \cap E_{+}} \frac{1}{\left|x^{*}-x_{0}\right|^{3+2 \alpha}} d x^{*}\right) \\
& =0 .
\end{aligned}
$$

Acknowledgments: The first author has been supported by GNAMPA (Gruppo Nazionale per l' Analisi Matematica, la Probabilità e loro Applicazioni), by the ERC starting grant EPSILON n 277749, by MINECO MTM2011-27739-C04-01 (Spain) and is part of the Catalan research group 2014 SGR 1083. The second and third authors have been supported by grants Fondecyt 1130360, 1150066, Fondo Basal CMM and Millenium Nucleus CAPDE NC130017.

\section{REFERENCES}

[1] G. Alberti, G. Bouchitté, and S. Seppecher, Phase transition with the line-tension effect, Arch. Rational Mech. Anal. 144 (1998), 1-46.

[2] C. Brändle, E. Colorado, A. de Pablo, and U. Sanchez, A concave-convex elliptic problem involving the fractional Laplacian. Proc. Roy. Soc. Edinburgh Sect. A 143 (2013), no. 1, 39-71.

[3] V. Banica, M.d.M. Gonzalez. and M. Saez, Some constructions for the fractional Laplacian on noncompact manifolds, Rev. Mat. Iberoam. 31 (2015), no. 2, 681-712.

[4] X. Cabré and E. Cinti, Energy estimates and 1-D symmetry for nonlinear equations involving the half-Laplacian. Discrete Contin. Dyn. Syst. 28 (2010), no. 3, 1179-1206.

[5] X. Cabré and E. Cinti, Sharp energy estimates for nonlinear fractional diffusion equations. Calc. Var. Partial Differential Equations 49 (2014), no. 1-2, 233-269.

[6] X. Cabré, M. Fall, and T. Weth, Delaunay hypersurfaces with constant nonlocal mean curvature, preprint. (Available at http://arxiv.org/abs/1602.02623). 
[7] X. Cabré, M. Fall, J. Solà-Morales, T. Weth, Curves and surfaces with constant nonlocal mean curvature: meeting Alexandrov and Delaunay, accepted for publication in J. Reine Angew. Math. (Available at http://arxiv.org/abs/1503.00469).

[8] X. Cabré and Y. Sire, Nonlinear equations for fractional Laplacians, I: Regularity, maximum principles, and Hamiltonian estimates, Ann. Inst. H. Poincaré Anal. Non Linéaire 31 (2014), no. 1, 23-53.

[9] X. Cabré and Y. Sire, Nonlinear equations for fractional Laplacians II: Existence, uniqueness, and qualitative properties of solutions, Trans. Amer. Math. Soc. 367 (2015), no. 2, 911-941

[10] X. Cabré and J. Solà-Morales, Layer solutions in a halph-space for boundary reactions, Comm. Pure and Appl. Math. 58 (2005), 1678-1732.

[11] X. Cabré and J. Tan, Positive solutions of nonlinear problems involving the square root of the Laplacian, Adv. Math. 224 (2010), no. 5, 2052-2093.

[12] L. Caffarelli, J-M. Roquejoffre, and O. Savin, Nonlocal minimal surfaces, Comm. Pure Appl. Math. 63 (2010), 1111-1144.

[13] L. Caffarelli and L. Silvestre, An extension problem related to the fractional Laplacian, Comm. Part. Diff. Eq. 32 (2007), 1245-1260.

[14] E. Cinti, Saddle-shaped solutions of bistable elliptic equations involving the halfLaplacian, Ann. Sc. Norm. Super. Pisa Cl. Sci. (5) 12, (2013), no. 3, 623-664.

[15] G. Ciraolo, A. Figalli, F. Maggi, M. Novaga, Rigidity and sharp stability estimates for hypersurfaces with constant and almost-constant nonlocal mean curvature, accepted for publication in J. Reine Angew. Math. .

[16] J. Davila, M. del Pino, S. Dipierro, E. Valdinoci, Nonlocal Delaunay surfaces, accepted for publication in Nonlinear Anal. .

[17] J. Davila, M. del Pino, and J. Wei, Nonlocal s-minimal surfaces and Lawson Cones, preprint. (Available at: http://arxiv.org/pdf/1402.4173.pdf).

[18] M. del Pino, M. Musso and F. Pacard, Solutions of the Allen-Cahn equation which are invariant under screw-motion, Manuscripta Math. 138, (2012), no. 3-4, 273-286.

[19] M.d.M. González, Gamma convergence of an energy functional related to the fractional Laplacian, Calc. Var. Part. Diff. Eq. 36 (2009), 173-210.

[20] L. Modica and S. Mortola, Un esempio di $\Gamma$-convergenza (Italian), Boll. Un. Mat. Ital. B (5) 14 (1977), no. 1, 285-299.

[21] G. Palatucci, O. Savin, and E. Valdinoci, Local and global minimizers for a variational energy involving a fractional norm, Ann. Mat. Pura Appl. (4) 192 (2013), no. 4, 673718.

[22] O. Savin and E. Valdinoci, Г-convergence for nonlocal phase transitions, Ann. Inst. H. Poincaré Anal. Non Linéaire 29, (2012), no. 4, 479-500.

[23] R. Servadei and E. Valdinoci, On the spectrum of two different fractional operators. Proc. Roy. Soc. Edinburgh Sect. A 144 (2014), no. 4, 831-855.

[24] J. Tan, Positive solutions for non local elliptic problems. Discrete Contin. Dyn. Syst. 33 (2013), no. 2, 837-859.

E.C., Weierstrass Institute for Applied Analysis and Stochastics, MohrenSTR. 39, 10117 BERLin (GERMANY)

E-mail address: cinti@wias-berlin.de

J. D. and M. D.P., Departamento de Ingeniería Matemática, Facultad de Ciencias Físicas y Matemáticas, Universidad de Chile. Casilla 170, Correo 3, Santiago, Chile.

E-mail address: jdavila@dim.uchile.cl

E-mail address: delpino@dim.uchile.cl 